Provided for non-commercial research and education use. Not for reproduction, distribution or commercial use.

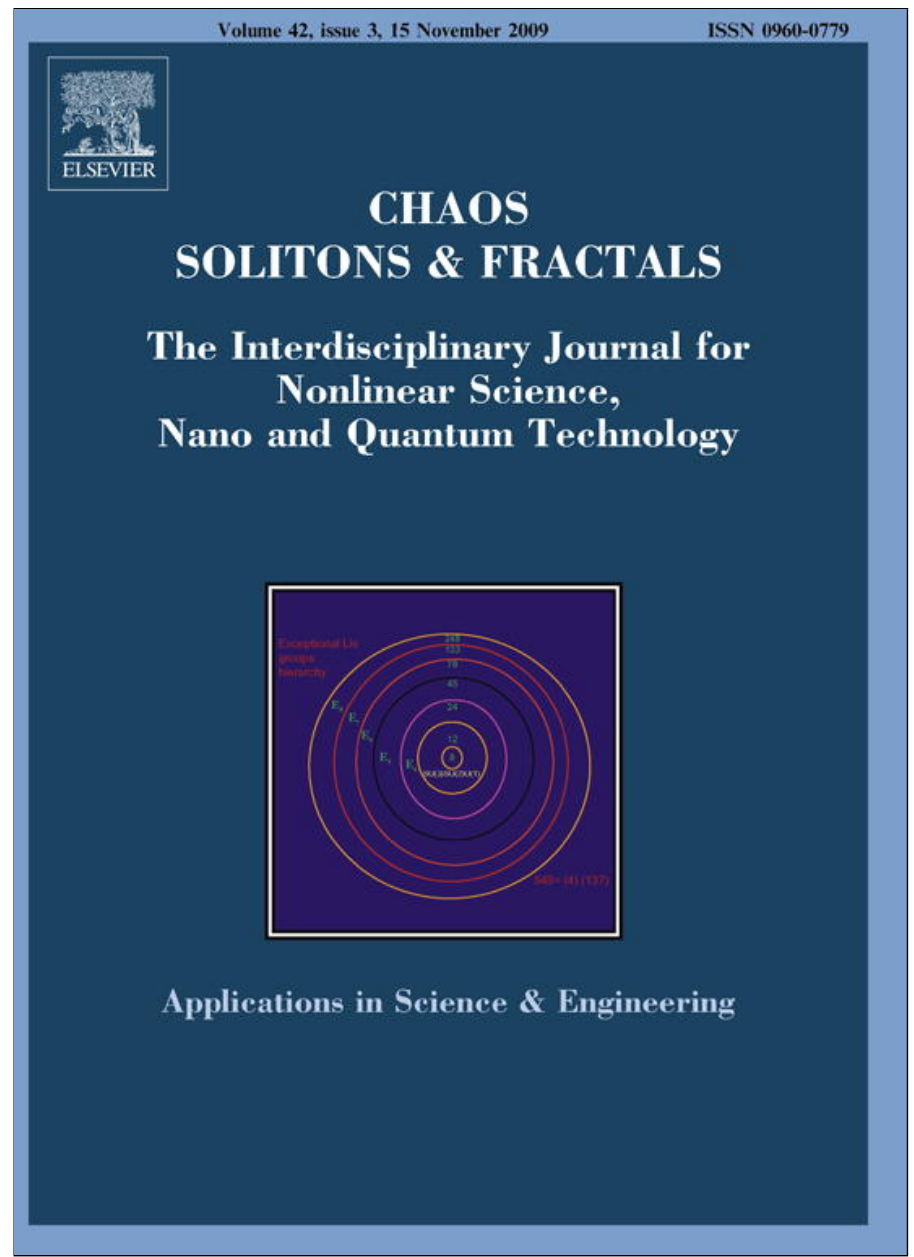

This article appeared in a journal published by Elsevier. The attached copy is furnished to the author for internal non-commercial research and education use, including for instruction at the authors institution and sharing with colleagues.

Other uses, including reproduction and distribution, or selling or licensing copies, or posting to personal, institutional or third party websites are prohibited.

In most cases authors are permitted to post their version of the article (e.g. in Word or Tex form) to their personal website or institutional repository. Authors requiring further information regarding Elsevier's archiving and manuscript policies are encouraged to visit:

http://www.elsevier.com/copyright 


\title{
Kneading theory analysis of the Duffing equation
}

\author{
Acilina Caneco ${ }^{\mathrm{a}, *}$, Clara Grácio ${ }^{\mathrm{b}}$, J. Leonel Rocha ${ }^{\mathrm{c}}$ \\ ${ }^{a}$ Mathematics Unit, DEETC, Instituto Superior de Engenharia de Lisboa Rua Conselheiro Emídio Navarro, 1, 1959-007 Lisboa, Portugal \\ bepartment of Mathematics, Universidade de Évora and CIMA-UE, Rua Romão Ramalho, 59, 7000-671 Évora, Portugal \\ c Mathematics Unit, DEQ Instituto Superior de Engenharia de Lisboa, Rua Conselheiro Emídio Navarro, 1, 1959-007 Lisboa, Portugal
}

\section{A R T I C L E I N F O}

Article history:

Accepted 11 March 2009

Dedicated to the memory of J. Sousa Ramos

\begin{abstract}
A B S T R A C T
The purpose of this paper is to study the symmetry effect on the kneading theory for symmetric unimodal maps and for symmetric bimodal maps. We obtain some properties about the kneading determinant for these maps, that implies some simplifications in the usual formula to compute, explicitly, the topological entropy. As an application, we study the chaotic behaviour of the two-well Duffing equation with forcing.
\end{abstract}

(c) 2009 Elsevier Ltd. All rights reserved.

\section{Motivation and introduction}

The Duffing equation has been used to model the nonlinear dynamics of special types of mechanical and electrical systems. This differential equation has been named after the studies of Duffing in 1918 [1], has a cubic nonlinearity and describes an oscillator. It is the simplest oscillator displaying catastrophic jumps of amplitude and phase when the frequency of the forcing term is taken as a gradually changing parameter. It has drawn extensive attention due to the richness of its chaotic behaviour with a variety of interesting bifurcations, torus and Arnold's tongues. The main applications have been in electronics, but it can also have applications in mechanics and in biology. For example, the brain is full of oscillators at micro and macro level [15]. There are applications in neurology, ecology, secure communications, cryptography, chaotic synchronization, and so on. Due to the rich behaviour of these equations, recently there has been also several studies on the synchronization of two coupled Duffing equations [13,14]. The most general forced form of the Duffing equation is $x^{\prime \prime}+a x^{\prime}+\left(\beta x^{3} \pm \omega_{0}^{2} x\right)=b \cos (\omega t+\phi)$. Depending on the parameters chosen, the equation can take a number of special forms. In [9,4], Mira et al. studied this equation with $\beta=1, \omega_{0}=0, \omega=1$ and $\phi=0$. They studied the bifurcations sets in the plane for different values of the parameter $a$, based on the notions of crossroad area, saddle area, spring area, island, lip and quasi-lip.

If the coefficient of $x$ is positive, this equation represents the single-well Duffing equation and if it is negative, we have the two-well Duffing equation. But there are also studies of a three-well Duffing system with two periodic forcings [3]. We will study the two-well equation with $\omega_{0}^{2}=1, \beta=1$ and $\phi=0$, i.e.,

$$
x^{\prime \prime}+a x^{\prime}+x^{3}-x=b \cos (\omega t) \text {. }
$$

In [2], Xie et al. used symbolic dynamics to study the behaviour of chaotic attractors and to analyze different periodic windows inside a closed bifurcation region in the parameter plane. Symbolic dynamics is a rigorous tool to understand chaotic motions in dynamical systems.

In this work, we will use techniques of kneading theory due essentially to Milnor and Thurston [8] and Sousa Ramos [57], applying these techniques to the study of the chaotic behaviour. We will use kneading theory to evaluate the topological entropy, which measures the chaoticity of the system. Generally, the graphic of the return map is a very complicated set of points. Therefore, in order to be able to apply these techniques, we show, in Section 2, regions in the parameter plane where

\footnotetext{
* Corresponding author.

E-mail addresses: acilina@deetc.isel.ipl.pt (A. Caneco), mgracio@uevora.pt (C. Grácio), jrocha@deq.isel.ipl.pt (J. Leonel Rocha).
} 
the first return Poincaré map is close to a one-dimensional object. Indeed, by numerical simulations, we found a region $\mathscr{U}$ where the return map is like a unimodal map and a region $\mathscr{B}$ where the return map is like a bimodal map, see Fig. 1 . Furthermore, we notice that some parameter values in region $\mathscr{U}$ correspond to pairs of unimodal maps that are symmetric (see Table 2). In the same way, some parameter values in region $\mathscr{B}$ correspond to symmetric bimodal maps. In fact, in Table 3 , we may see two kinds of symmetric bimodal map. In Section 3, we briefly describe the kneading theory for a general $m$-modal map and then we study the effects of symmetry on the kneading determinant for symmetric unimodal maps and for symmetric bimodal maps. It is well known that symmetry is an important issue and has been intensively studied [11,12]. We prove that the kneading matrix present some simplifications due to this symmetries. Examples are given, in Section 4 , for the Duffing equation, but this method can be applied to others equations, whose return maps have this kind of symmetry.

\section{Poincaré maps, bifurcation diagrams, unimodal and bimodal regions}

We choose the Poincaré section defined by the plane $y=0$, since it is transversal to the flow, it contains all fixed points and captures most of the interesting dynamics. For our choice of the Poincare section, the parameterization is simply realized by the $x$ coordinates of the points. In terms of these $\left\{x_{i}\right\}$, a first return Poincaré map $x_{n} \rightarrow x_{n+1}$ is constructed. This will be done for each choice of the parameters $a, b$ and $w$. In the examples presented in Section 4 , we will fix the parameter $w=1.18$ and we will study the first return Poincare map for different values of the parameters $(a, b)$. We will denote this Poincaré map by $f_{a, b}$. In order to see how the first return Poincaré map change with the parameters, we make bifurcation diagrams. For example, in Fig. 2, we plot the variation of the first coordinate of the first return Poincaré map, $x_{n}$, versus the parameter $b \in[0.15,0.5]$, for a fixed value $a=0.25$. We see clearly the growing of complexity as the parameter $b$ increases.

To have a more precise notion of this complexity, we may compute the topological entropy of the first return Poincaré map in each region $\mathscr{U}$ and $\mathscr{B}$. Let us first look, in the parameter plane $(a, b)$, for those regions where the first return Poincaré map behaves like a unimodal or a bimodal map, because we have an explicit way to evaluate the topological entropy in those cases. For example, for $a=0.25$, we found that when $b \in[0.254,0.260]$ the first return Poincaré map $f_{a, b}$ behaves like a unimodal map and when $b \in[0.479,0.483]$ the first return Poincaré map $f_{a, b}$ behaves like a bimodal map. These range of values correspond to two narrow vertical bands in the bifurcation diagram in Fig. 1.

\section{Kneading theory and topological entropy}

Consider a compact interval $I \subset \mathbb{R}$ and a $m$-modal map $f: I \rightarrow I$, i.e., the map $f$ is piecewise monotone, with $m$ critical points and $m+1$ subintervals of monotonicity. Suppose $I=\left[c_{0}, c_{m+1}\right]$ can be divided by a partition of points $\mathscr{P}=\left\{c_{0}, c_{1}, \ldots, c_{m+1}\right\}$ in a finite number of subintervals $I_{1}=\left[c_{0}, c_{1}\right], I_{2}=\left[c_{1}, c_{2}\right], \ldots, I_{m+1}=\left[c_{m}, c_{m+1}\right]$, in such a way that the restriction of $f$ to each interval $I_{j}$ is strictly monotone, either increasing or decreasing. Assuming that each interval $I_{j}$ is the maximal interval where the function is strictly monotone, these intervals $I_{j}$ are called laps of $f$ and the number of distinct laps is called the lap number, $\ell$, of $f$. In the interior of the interval $I$, the points $c_{1}, c_{2}, \ldots, c_{m}$ are local minimum or local maximum of $f$ and are called turning or critical points of the function. The limit of the $n$-root of the lap number of $f^{n}$ (where $f^{n}$ denotes the composition of $f$ with itself $n$ times) is called the growth number of $f$, i.e., $s=\lim _{n \rightarrow \infty} \sqrt[n]{\ell\left(f^{n}\right)}$. In [10], Misiurewicz and Szlenk define the topological entropy as the logarithm of the growth number $h_{\text {top }}(f)=\log s$. In [8], Milnor and Thurston developed the concept of kneading determinant, denoted by $D(t)$, as a formal power series from which we can compute the topological entropy as the logarithm of the inverse of its minimum real positive root. On the other hand, Sousa Ramos et al., using homological properties proved a precise relation between the kneading determinant and the characteristic polynomial of the Markov transition matrix associated with the itinerary of the critical points. In fact, they proved that the topological entropy is the logarithm of the spectral radius of this matrix.

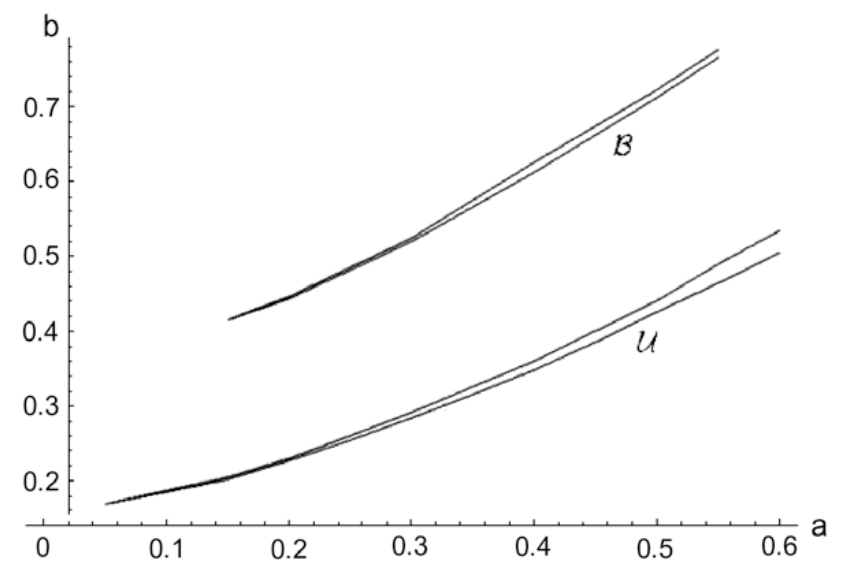

Fig. 1. Unimodal region $(\mathscr{U})$ and bimodal region $(\mathscr{B})$. 


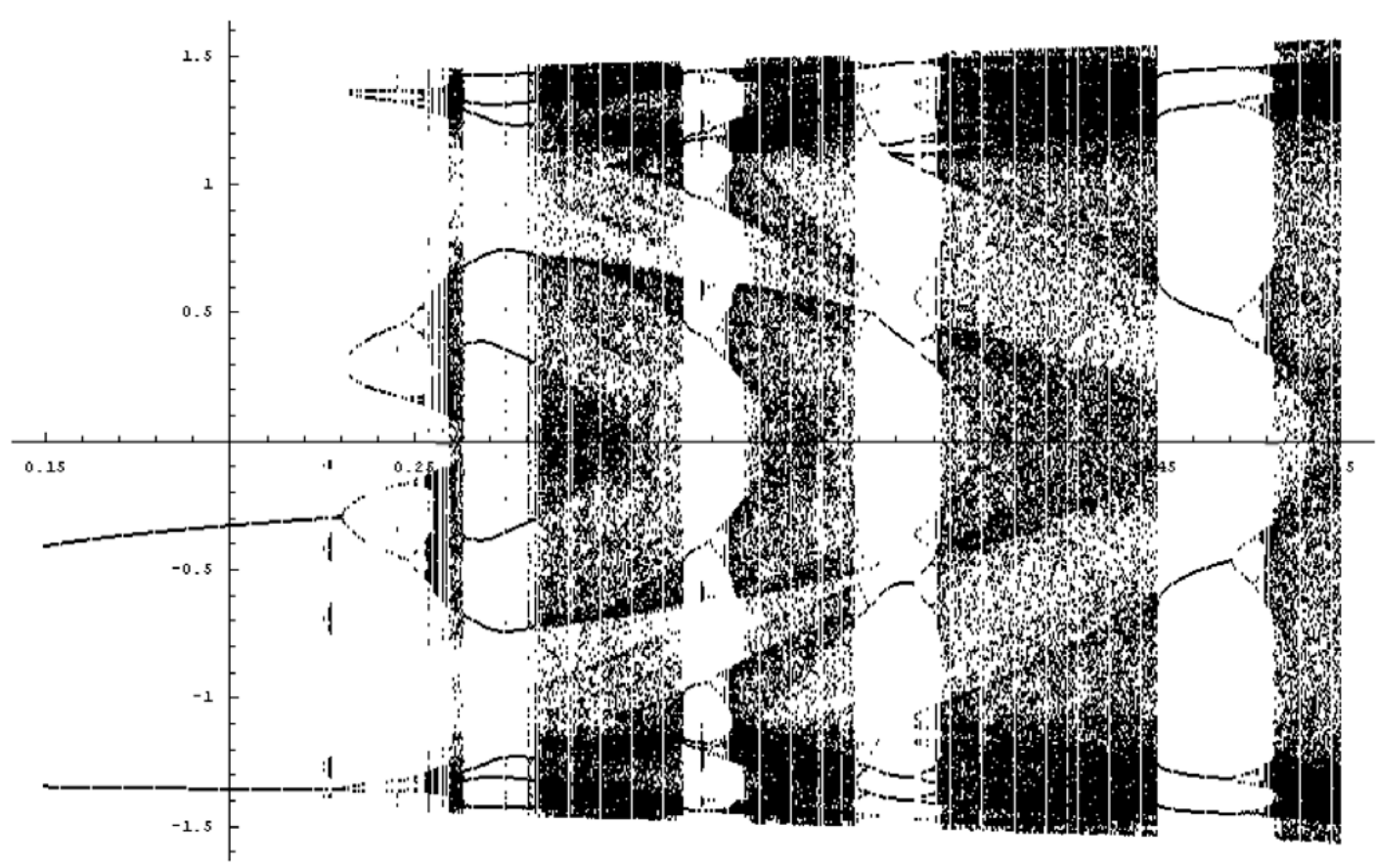

Fig. 2. Bifurcation diagram for $x_{n}$ as a function of $b$ with $a=0.25$ and $b \in[0.15,0.5]$.

The intervals $I_{j}=\left[c_{j-1}, c_{j}\right]$ are separated by the critical points, numbered by its natural order $c_{1}<c_{2}<\cdots<c_{m}$. We compute the images by $f, f^{2}, \ldots, f^{n}, \ldots$ of a critical point $c_{j}(j=1, \ldots, m-1)$ and we obtain its orbit

$$
O\left(c_{j}\right)=\left\{c_{j}^{n}: c_{j}^{n}=f^{n}\left(c_{j}\right), n \in \mathbb{N}\right\} .
$$

If $f^{n}\left(c_{j}\right)$ belongs to an open interval $\left.I_{k}=\right] c_{k-1}, c_{k}\left[\right.$, then we associate to it a symbol $L_{k}$ with $k=1, \ldots, m+1$. If there is an $r$ such that $f^{n}\left(c_{j}\right)=c_{r}$, with $r=1, \ldots, m$, then we associate to it the symbol $A_{r}$. So, to each point $c_{j}$, we associate a symbolic sequence, called the address of $f^{n}\left(c_{j}\right)$, denoted by $S=S_{1} S_{2} \cdots S_{n} \cdots$, where the symbols $S_{k}$ belong to the $m$-modal alphabet, with $2 m+1$ symbols, i.e., $\mathscr{A}_{m}=\left\{L_{1}, A_{1}, L_{2}, A_{2}, \ldots, A_{m}, L_{m+1}\right\}$. The symbolic sequence $S=S_{0} S_{1} S_{2} \cdots S_{n} \cdots$ can be periodic, eventually periodic or aperiodic [7]. The address of a critical point $c_{j}$ is said eventually periodic if there is a number $p \in \mathbb{N}$, such that the address of $f^{n}\left(c_{j}\right)$ is equal to the address of $f^{n+p}\left(c_{j}\right)$, for large $n \in \mathbb{N}$. The smallest of such $p$ is called the eventual period.

To each symbol $L_{k} \in \mathscr{A}_{m}$, with $k=1, \ldots, m+1$, define its sign by

$$
\varepsilon\left(L_{k}\right)= \begin{cases}-1 & \text { if } f \text { is decreasing in } I_{k} \\ 1 & \text { if } f \text { is increasing in } I_{k}\end{cases}
$$

and $\varepsilon\left(A_{k}\right)=0$, with $k=1, \ldots, m$. We can compute the numbers $\tau_{k}=\prod_{i=0}^{k-1} \varepsilon\left(L_{k}\right)$ for $k>0$, and take $\tau_{0}=1$. The invariant coordinate of the symbolic sequence $S$, associated with a critical point $c_{j}$, is defined as the formal power series

$$
\theta_{c_{j}}(t)=\sum_{k=0}^{k=\infty} \tau_{k} t^{k} S_{k} .
$$

The kneading increments of each critical point $c_{j}$ are defined by

$$
v_{c_{j}}(t)=\theta_{c_{j}^{+}}(t)-\theta_{c_{j}^{-}}(t) \quad \text { with } j=1, \ldots m,
$$

where $\theta_{c_{ \pm}^{ \pm}}(t)=\lim _{x \rightarrow c_{1}^{ \pm}} \theta_{x}(t)$. Separating the terms associated with the symbols $L_{1}, L_{2}, \ldots, L_{m+1}$ of the alphabet $\mathscr{A}_{m}$, the increments $v_{j}(t)$, are written in the form

$$
v_{c_{j}}(t)=N_{j 1}(t) L_{1}+N_{j 2}(t) L_{2}+\cdots+N_{j(m+1)}(t) L_{m+1} .
$$

The coefficients $N_{j k}$ in the ring $Z[[t]]$ are the entries of the $m \times(m+1)$ kneading matrix

$$
N(t)=\left[\begin{array}{ccc}
N_{11}(t) & \cdots & N_{1(m+1)}(t) \\
\vdots & \ddots & \vdots \\
N_{m 1}(t) & \cdots & N_{m(m+1)}(t)
\end{array}\right] .
$$

From this matrix, we compute the determinants $D_{j}(t)=\operatorname{det} \widehat{N}(t)$, where $\widehat{N}(t)$ is obtained from $N(t)$ removing the $j$ column $(j=1, \ldots, m+1)$, and 


$$
D(t)=\frac{(-1)^{j+1} D_{j}(t)}{1-\varepsilon\left(L_{j}\right) t}
$$

is called the kneading determinant. Here, $\varepsilon\left(L_{j}\right)$ is defined like in (2).

Let $f$ be a $m$-modal map and $D(t)$ defined as above. Let $s$ be the growth number of $f$, then the topological entropy of the $\operatorname{map} f$ is, see [8],

$$
h_{\text {top }}(f)=\log s \text { with } s=\frac{1}{t^{*}} \quad \text { and } \quad t^{*}=\min \{t \in[0,1]: D(t)=0\} .
$$

\subsection{Symmetry effect on the kneading theory for unimodal maps}

To each value of the parameters $(a, b)$, consider a function $f_{a, b}: I \rightarrow I$, from the compact interval $I \subset \mathbb{R}$ to itself, such that $I=\left[c_{0}, c_{1}\right]$ is divided in two subintervals $L=\left[c_{0}, c\right]$ and $R=\left[c, c_{1}\right]$, where $c$ denote the single critical point of $f_{a, b}$ and suppose that $\left.f_{a, b}\right|_{L}$, and $\left.f_{a, b}\right|_{R}$ are monotone functions. Such a map is called a unimodal map. With the above procedure, we can compute the topological entropy for the unimodal maps. In Table 1, we show the kneading data and the topological entropy associated to unimodal first return Poincaré map $f_{a, b}$, obtained for some values of the parameters $(a, b) \in \mathscr{U}$ and $w=1.18$. Note that some sequences of Table 1 have zero topological entropy. They have periods power of two, so this result, obtained by numerical computation, was expected as a consequence of Sharkovsky theorem. Note also, that some sequences of Table 1 are eventually periodic, see [7]. Looking at Table 1, we notice that there are pairs of maps $f_{a, b}$ and $f_{a_{1}, b_{1}}$ with kneading sequences such that we can obtain one of them by changing the symbols $L$ by $R$ and $R$ by $L$. They are somehow symmetric and they have the same topological entropy. In Table 2, we have some pairs of symmetric unimodal maps, chosen from Table 1. We wonder if this equality between entropies holds for all symmetric unimodal maps. In order to prove that, we must define more precisely what we mean by mirror symmetric unimodal maps.

Definition 1. Let $f_{a, b}$ and $f_{a_{1}, b_{1}}$ be two unimodal maps. Assume that $f_{a, b}$ has a critical point $c_{1}$ and has a periodic kneading sequence $\left(A S_{1} S_{2} \cdots S_{p-1}\right)^{\infty}$, being $A$ the symbol corresponding to $c_{1}$. Then, we say that $f_{a_{1}, b_{1}}$, with a critical point $c_{2}$, is the mirror symmetric map of $f_{a, b}$ if and only if $f_{a_{1}, b_{1}}$ has the kneading sequence $\left(B \widehat{S}_{1} \widehat{S}_{2} \ldots \widehat{S}_{p-1}\right)^{\infty}$, such that

$$
\left\{\begin{array} { l } 
{ c _ { 2 } \text { is a minimum if } c _ { 1 } \text { is a maximum } } \\
{ c _ { 2 } \text { is a maximum if } c _ { 1 } \text { is a minimum } }
\end{array} \text { and } \left\{\begin{array}{ll}
\widehat{S}_{j}=R & \text { if } S_{j}=L, \\
\widehat{S}_{j}=L & \text { if } S_{j}=R,
\end{array}\right.\right.
$$

where $B$ is the symbol corresponding to $c_{2}$. In that case, we call to (9) a mirror transformation to the unimodal maps $f_{a, b}$ and $f_{a_{1}, b_{1}}$.

Lemma 2. If $f_{a, b}$ and $f_{a_{1}, b_{1}}$ are two mirror symmetric unimodal maps, in the sense of (9), then

$$
N_{11_{f_{a, b}}}(t)=-N_{12_{f_{1}, b_{1}}}(t) \text { and } N_{12_{f_{a, b}}}(t)=-N_{11_{f_{a_{1}, b_{1}}}}(t) .
$$

\begin{tabular}{|c|c|c|}
\hline$(a, b)$ & Kneading data for $f_{a, b}$ & $h_{\text {top }}\left(f_{a, b}\right)$ \\
\hline$(0.40000,0.34840)$ & $(C L R L L L R L)^{\infty}$ & 0.0 \\
\hline$(0.40000,0.34850)$ & $(C L R L L L R L)^{\infty}$ & 0.0 \\
\hline$(0.40000,0.35000)$ & $(C L R L L L R L)^{\infty}$ & 0.0 \\
\hline$(0.40000,0.35100)$ & $(C L R L L L R L R L R L)^{\infty}$ & $0.12030 \ldots$ \\
\hline$(0.40000,0.35200)$ & $(C L R L L L R L R L R L)^{\infty}$ & $0.12030 \ldots$ \\
\hline$(0.40000,0.35300)$ & $\operatorname{CLRLL}(L R)^{\infty}$ & 0.17329 \\
\hline$(0.40000,0.35500)$ & $\operatorname{CLRLL}(L R)^{\infty}$ & 0.17329. \\
\hline$(0.40000,0.35600)$ & $\operatorname{CLRLL}(\operatorname{LRLR})^{\infty}$ & 0.17329. \\
\hline$(0.40000,0.35700)$ & $(C L R L L L R L R L)^{\infty}$ & $0.20701 \ldots$ \\
\hline$(0.40000,0.35730)$ & $(C L R L L L R L R L)^{\infty}$ & $0.20701 \ldots$ \\
\hline$(0.40000,0.35780)$ & $(C L R L L L)^{\infty}$ & $0.24061 \ldots$ \\
\hline$(0.40000,0.35810)$ & $(C L R L L L)^{\infty}$ & $0.24061 \ldots$ \\
\hline$(0.29540,0.28100)$ & $(C L R L L L R L)^{\infty}$ & 0.0 \\
\hline$(0.29540,0.28115)$ & $(C L R L L L R L)^{\infty}$ & 0.0 \\
\hline$(0.29540,0.28520)$ & $(C R L R R R L)^{\infty}$ & 0.17329. \\
\hline$(0.29540,0.28628)$ & $C R(L R R R)^{\infty}$ & $0.24061 \ldots$ \\
\hline$(0.29540,0.28750)$ & $(C R L R R R)^{\infty}$ & $0.24061 \ldots$ \\
\hline$(0.30000,0.28620)$ & $(C L R L L L)^{\infty}$ & $0.24061 \ldots$ \\
\hline$(0.30000,0.28580)$ & $C R(L R R R)^{\infty}$ & $0.24061 \ldots$ \\
\hline$(0.20000,0.22900)$ & $(C L R L L L R L)^{\infty}$ & 0.0 \\
\hline$(0.20000,0.22920)$ & $(C R L R R R L R)^{\infty}$ & 0.0 \\
\hline$(0.25000,0.25390)$ & $(C L R L L L R L L L R L L L R L)^{\infty}$ & 0.0 \\
\hline$(0.25000,0.25520)$ & $(C L R L L L R L)^{\infty}$ & 0.0 \\
\hline$(0.25000,0.25870)$ & $(C L R L L L)^{\infty}$ & $0.24061 \ldots$ \\
\hline
\end{tabular}

Table 1

Kneading data and topological entropy for some unimodal maps. 
Table 2

Some pairs of parameter values corresponding to symmetric unimodal maps.

\begin{tabular}{lll}
\hline$(a, b)$ & $\left(a_{1}, b_{1}\right)$ & \\
\hline$(0.40000,0.34840)$ & $(0.20000,0.22920)$ & $h_{\text {top }}$ \\
$(0.20000,0.22920)$ & $(0.20000,0.22900)$ & 0 \\
$(0.20000,0.25520)$ & $(0.20000,0.22920)$ & 0 \\
$(0.40000,0.35300)$ & $(0.29540,0.28520)$ & 0 \\
$(0.40000,0.35780)$ & $(0.29540,0.28750)$ & $0.17329 \ldots$ \\
$(0.29540,0.28750)$ & $(0.40000,0.35810)$ & $0.24061 \ldots$ \\
$(0.30000,0.28620)$ & $(0.29540,0.28750)$ & $0.24061 \ldots$ \\
$(0.25000,0.25870)$ & $(0.29540,0.28750)$ & $0.24061 \ldots$ \\
\hline
\end{tabular}

Proof. Let $\left(A S_{1} S_{2} \cdots S_{p-1}\right)^{\infty}$ and $\left(B \widehat{S}_{1} \widehat{S}_{2} \cdots \widehat{S}_{p-1}\right)^{\infty}$ be the kneading sequences of $f_{a, b}$ and $f_{a_{1}, b_{1}}$, respectively, where

$$
\begin{array}{ll}
c_{1}^{+} \rightarrow\left(R S_{1} S_{2} \cdots S_{p-1}\right)^{\infty}, & c_{2}^{+} \rightarrow\left(R \widehat{S}_{1} \widehat{S}_{2} \cdots \widehat{S}_{p-1}\right)^{\infty}, \\
c_{1}^{-} \rightarrow\left(L S_{1} S_{2} \cdots S_{p-1}\right)^{\infty}, & c_{2}^{-} \rightarrow\left(L \widehat{S}_{1} \widehat{S}_{2} \cdots \widehat{S}_{p-1}\right)^{\infty} .
\end{array}
$$

The invariant coordinates of the sequence associated to the critical point of $f_{a, b}$ and $f_{a_{1}, b_{1}}$ are, respectively,

$$
\begin{aligned}
& \theta_{c_{1}^{+}}(t)=\sum_{k=0}^{k=\infty} \tau_{k}\left(c_{1}^{+}\right) t^{k} S_{k}=\left(R+\sum_{k=1}^{k=p-1} \tau_{k}\left(c_{1}^{+}\right) t^{k} S_{k}\right) \frac{1}{1-\tau_{p}\left(c_{1}^{+}\right) t^{p}}, \\
& \theta_{c_{1}^{-}}(t)=\sum_{k=0}^{k=\infty} \tau_{k}\left(c_{1}^{-}\right) t^{k} S_{k}=\left(L+\sum_{k=1}^{k=p-1} \tau_{k}\left(c_{1}^{-}\right) t^{k} S_{k}\right) \frac{1}{1-\tau_{p}\left(c_{1}^{-}\right) t^{p}}, \\
& \theta_{c_{2}^{+}}(t)=\sum_{k=0}^{k=\infty} \tau_{k}\left(c_{2}^{+}\right) t^{k} \widehat{S}_{k}=\left(R+\sum_{k=1}^{k=p-1} \tau_{k}\left(c_{2}^{+}\right) t^{k} \widehat{S}_{k}\right) \frac{1}{1-\tau_{p}\left(c_{2}^{+}\right) t^{p}}, \\
& \theta_{c_{2}^{-}}(t)=\sum_{k=0}^{k=\infty} \tau_{k}\left(c_{2}^{-}\right) t^{k} \widehat{S}_{k}=\left(L+\sum_{k=1}^{k=p-1} \tau_{k}\left(c_{2}^{-}\right) t^{k} \widehat{S}_{k}\right) \frac{1}{1-\tau_{p}\left(c_{2}^{-}\right) t^{p}} .
\end{aligned}
$$

Note that, $\tau_{p}\left(c_{1}^{-}\right)=\tau_{p}\left(c_{2}^{+}\right)=-\tau_{p}\left(c_{1}^{+}\right)=-\tau_{p}\left(c_{2}^{-}\right)$, because $\varepsilon\left(S_{k}\right)=\varepsilon\left(\widehat{S}_{k}\right)$ and due to (9). Denoting by

$$
L_{k}\left(c_{j}^{ \pm}\right)=\sum_{\substack{i=1 \\ S_{i} \text { or } \widehat{S}_{i}=L}}^{k-1} \tau_{i}\left(c_{j}^{ \pm}\right) t^{i} \text { and } R_{k}\left(c_{j}^{ \pm}\right)=\sum_{\substack{i=1 \\ S_{i} \text { or } \widehat{S}_{i}=R}}^{k-1} \tau_{i}\left(c_{j}^{ \pm}\right) t^{i}
$$

with $j=1,2$, we notice that $L_{p}\left(c_{1}^{ \pm}\right)=R_{p}\left(c_{2}^{\mp}\right)$ and $R_{p}\left(c_{1}^{ \pm}\right)=L_{p}\left(c_{2}^{\mp}\right)$, because the number of symbols $L$ in $c_{1}^{+}$is equal to the number of $R$ in $c_{2}^{-}$and the number of symbols $R$ in $c_{1}^{+}$is equal to the number of $L$ in $c_{2}^{-}$. Separating the terms associated with the symbols $L$ and $R$ of the alphabet $\mathscr{A}=\{A, L, R\}$, we may write the invariant coordinates in the following way:

$$
\begin{aligned}
& \theta_{c_{1}^{+}}(t)=\left[R+L_{p}\left(c_{1}^{+}\right) L+R_{p}\left(c_{1}^{+}\right) R\right] \frac{1}{1-\tau_{p}\left(c_{1}^{+}\right) t^{p}}, \\
& \theta_{c_{1}^{-}}(t)=\left[L+L_{p}\left(c_{1}^{-}\right) L+R_{p}\left(c_{1}^{-}\right) R\right] \frac{1}{1+\tau_{p}\left(c_{1}^{+}\right) t^{p}}
\end{aligned}
$$

and

$$
\begin{aligned}
& \theta_{c_{2}^{+}}(t)=\left[R+R_{p}\left(c_{1}^{-}\right) L+L_{p}\left(c_{1}^{-}\right) R\right] \frac{1}{1+\tau_{p}\left(c_{1}^{+}\right) t^{p}}, \\
& \theta_{c_{2}^{-}}(t)=\left[L+R_{p}\left(c_{1}^{+}\right) L+L_{p}\left(c_{1}^{+}\right) R\right] \frac{1}{1-\tau_{p}\left(c_{1}^{+}\right) t^{p}} .
\end{aligned}
$$

Consequently, the entries of the kneading increments for the points $c_{1}$ and $c_{2}$ are

$$
N_{11_{f_{a, b}}}(t)=\frac{L_{p}\left(c_{1}^{+}\right)\left[1+\tau_{p}\left(c_{1}^{+}\right) t^{p}\right]-\left[1+L_{p}\left(c_{1}^{-}\right)\right]\left[1-\tau_{p}\left(c_{1}^{+}\right) t^{p}\right]}{1-\tau_{p}^{2}\left(c_{1}^{+}\right) t^{2 p}}
$$

and

$$
N_{12_{f_{a, b}}}(t)=\frac{\left[1+R_{p}\left(c_{1}^{+}\right)\right]\left[1+\tau_{p}\left(c_{1}^{+}\right) t^{p}\right]-R_{p}\left(c_{1}^{-}\right)\left[1-\tau_{p}\left(c_{1}^{+}\right) t^{p}\right]}{1-\tau_{p}^{2}\left(c_{1}^{+}\right) t^{2 p}}
$$

It follows, as desired, that $N_{11_{f_{a, b}}}(t)=-N_{12_{f_{1}, b}}(t)$ and $N_{12_{f_{a, b}}}(t)=-N_{11_{f_{a_{1}, b_{1}}}}(t)$. 
The above lemma suggests the next result.

Proposition 3. The mirror symmetric unimodal maps $f_{a, b}$ and $f_{a_{1}, b_{1}}$, under the conditions of the previous lemma, have the same topological entropy.

Proof. This statement is a consequence of Lemma 2, i.e.,

$$
D_{1_{f_{a, b}}}(t)=N_{12 f_{a, b}}(t)=-N_{11_{f_{a_{1}, b}}}(t)=-D_{2_{f_{1}, b_{1}}}(t)
$$

and

$$
D_{2_{a, b}}(t)=N_{11 f_{a, b}}(t)=-N_{12_{f_{1}, b_{1}}}(t)=-D_{1_{f_{1}, b_{1}}}(t) .
$$

By (7) and noticing that $\varepsilon\left(L_{f_{a, b}}\right)=-\varepsilon\left(L_{f_{a_{1}, b_{1}}}\right)$, we have

$$
\begin{aligned}
D_{f_{a, b}}(t) & =\frac{D_{1_{f_{a, b}}}(t)}{1-\varepsilon\left(L_{f_{a, b}}\right) t}=-\frac{D_{2_{f_{a, b}}}(t)}{1+\varepsilon\left(L_{f_{a, b}}\right) t} \\
& =-\frac{D_{2_{f_{a_{1}, b_{1}}}}(t)}{1+\varepsilon\left(L_{f_{a_{1}, b}}\right) t}=\frac{D_{1_{f_{a_{1}, b_{1}}}}(t)}{1-\varepsilon\left(L_{f_{a_{1}, b}}\right) t}=D_{f_{a_{1}, b_{1}}}(t) .
\end{aligned}
$$

So, the maps $f_{a, b}$ and $f_{a_{1}, b_{1}}$ have the same kneading determinant $D(t)$ and, consequently, they have the same topological entropy.

\subsection{Symmetry effect on the kneading theory for bimodal maps}

To each value of the parameters $(a, b)$, consider a function $f_{a, b}: I \rightarrow I$, from the closed interval $I$ to itself, such that $I$ is divided in three subintervals $L=\left[c_{0}, c_{1}\right], M=\left[c_{1}, c_{2}\right]$ and $R=\left[c_{2}, c_{3}\right]$, where $c_{1}$ and $c_{2}$ denote the critical points of $f_{a, b}$ and suppose that $\left.f_{a, b}\right|_{L},\left.f_{a, b}\right|_{M}$ and $\left.f_{a, b}\right|_{R}$ are monotone functions. Such a map is called a bimodal map. For a bimodal map, the symbolic sequences corresponding to periodic orbits of the critical points $c_{1}$, with period $p$, and $c_{2}$ with period $k$, may be written as

$$
\left(\left(A S_{1} S_{2} \cdots S_{p-1}\right)^{\infty},\left(B Q_{1} \cdots Q_{k-1}\right)^{\infty}\right) .
$$

In this case, we have two periodic orbits, but in other cases of bimodal maps we have a single periodic orbit, of period $p+k$, that passes through both critical points (bistable case), for which we write only

$$
\left(A P_{1} \cdots P_{p-1} B Q_{1} \cdots Q_{k-1}\right)^{\infty} \text {. }
$$

See some examples in Table 3.

Now, we will study these two cases, with the additional condition that $p=k$ and the symbols $Q_{j}$ are the symmetric of the symbols $P_{j}$, in the sense of the following definition.

Definition 4. Let $f_{a, b}$ be a symmetric bimodal map for which the periodic kneading sequence, with period $q=2 p$, is

$$
S=\left(\left(A S_{1} S_{2} \cdots S_{p-1}\right)^{\infty},\left(B \widehat{S}_{1} \widehat{S}_{2} \cdots \widehat{S}_{p-1}\right)^{\infty}\right)
$$

or

$$
S=\left(A S_{1} S_{2} \cdots S_{p-1} B \widehat{S}_{1} \widehat{S}_{2} \cdots \widehat{S}_{p-1}\right)^{\infty}
$$

\begin{tabular}{|c|c|}
\hline$(a, b)$ & Kneading data for $f_{a, b}$ \\
\hline$(0.40000,0.61320)$ & $\left((A L R M R L)^{\infty},(B R L M L R)^{\infty}\right)$ \\
\hline$(0.40000,0.61420)$ & $(A L R M R L L B R L M L R R)^{\infty}$ \\
\hline$(0.40000,0.61500)$ & $(\text { ALRMRLLLRMRLMLRBRLMLRRRLMLRMRL) })^{\infty}$ \\
\hline$(0.40000,0.61600)$ & $(A L R M R L L L R B R L M L R R R L)^{\infty}$ \\
\hline$(0.40000,0.61900)$ & $A L R(M R L L L R B R L M L R R)^{\infty}$ \\
\hline$(0.40000,0.62350)$ & $(A L R(M R L L), B R L(M L R R))^{\infty}$ \\
\hline$(0.40000,0.62300)$ & $(A L R(M R L L), B R L(M L R R))^{\infty}$ \\
\hline
\end{tabular}

with $S_{1}, S_{2}, \ldots, S_{p-1} \in\{L, M, R\}$, such that

$$
\left\{\begin{array}{ll}
\widehat{S}_{j}=R & \text { if } S_{j}=L \\
\widehat{S}_{j}=L & \text { if } S_{j}=R \\
\widehat{S}_{j}=M & \text { if } S_{j}=M
\end{array} \text { and } \quad A \leftrightarrow B .\right.
$$

Table 3

Kneading data for some bimodal maps. 
The bimodal map $f_{a, b}$ is called a symmetric bimodal map and (12) is called a mirror transformation for this map.

We call $\widehat{S}_{j}$ the mirror image of $S_{j}$. The second half of $S$ is obtained from the first one by the mirror transformation, see [2]. Note that, this definition of symmetry is just for one bimodal map, while in Section 3.1 we defined symmetry between two different unimodal maps.

In the next results, we will find some interesting properties of the kneading theory due to this symmetry.

Lemma 5. Let $S=\left(\left(A S_{1} S_{2} \cdots S_{p-1}\right)^{\infty},\left(B \widehat{S}_{1} \widehat{S}_{2} \cdots \widehat{S}_{p-1}\right)^{\infty}\right)$ be a symmetric bimodal kneading sequence satisfying the mirror transformation (12). Then, we have

$$
D_{1}(t)=D_{3}(t)=N_{12}(t)\left(N_{13}(t)-N_{11}(t)\right) \quad \text { and } \quad D_{2}(t)=N_{13}^{2}(t)-N_{11}^{2}(t) .
$$

Proof. Set

$$
\begin{aligned}
& c_{1}^{+} \rightarrow\left(M S_{1} S_{2} \cdots S_{p-1}\right)^{\infty}, \quad c_{2}^{+} \rightarrow\left(R \widehat{S}_{1} \widehat{S}_{2} \cdots \widehat{S}_{p-1}\right)^{\infty}, \\
& c_{1}^{-} \rightarrow\left(L S_{1} S_{2} \cdots S_{p-1}\right)^{\infty}, \quad c_{2}^{-} \rightarrow\left(M \widehat{S}_{1} \widehat{S}_{2} \cdots \widehat{S}_{p-1}\right)^{\infty} .
\end{aligned}
$$

The invariant coordinates of the sequences associated to the critical points of $f_{a, b}$ are

$$
\begin{aligned}
& \theta_{c_{1}^{+}}(t)=\sum_{k=0}^{k=\infty} \tau_{k}\left(c_{1}^{+}\right) t^{k} S_{k}=\left(M+\sum_{k=1}^{k=p-1} \tau_{k}\left(c_{1}^{+}\right) t^{k} S_{k}\right) \frac{1}{1-\tau_{p}\left(c_{1}^{+}\right) t^{p}}, \\
& \theta_{c_{1}^{-}}(t)=\sum_{k=0}^{k=\infty} \tau_{k}\left(c_{1}^{-}\right) t^{k} S_{k}=\left(L+\sum_{k=1}^{k=p-1} \tau_{k}\left(c_{1}^{-}\right) t^{k} S_{k}\right) \frac{1}{1-\tau_{p}\left(c_{1}^{-}\right) t^{p}}, \\
& \theta_{c_{2}^{+}}(t)=\sum_{k=0}^{k=\infty} \tau_{k}\left(c_{2}^{+}\right) t^{k} \widehat{S}_{k}=\left(R+\sum_{k=1}^{k=p-1} \tau_{k}\left(c_{2}^{+}\right) t^{k} \widehat{S}_{k}\right) \frac{1}{1-\tau_{p}\left(c_{2}^{+}\right) t^{p}}, \\
& \theta_{c_{2}^{-}}(t)=\sum_{k=0}^{k=\infty} \tau_{k}\left(c_{2}^{-}\right) t^{k} \widehat{S}_{k}=\left(M+\sum_{k=1}^{k=p-1} \tau_{k}\left(c_{2}^{-}\right) t^{k} \widehat{S}_{k}\right) \frac{1}{1-\tau_{p}\left(c_{2}^{-}\right) t^{p}} .
\end{aligned}
$$

Note that, $\tau_{p}\left(c_{1}^{-}\right)=\tau_{p}\left(c_{2}^{+}\right)=-\tau_{p}\left(c_{1}^{+}\right)=-\tau_{p}\left(c_{2}^{-}\right)$, because $\varepsilon\left(S_{k}\right)=\varepsilon\left(\widehat{S}_{k}\right)$ and due to (12). Denoting by

$$
\begin{gathered}
L_{k}\left(c_{j}^{ \pm}\right)=\sum_{\substack{i=1 \\
S_{i} \text { or } \widehat{S}_{i}=L}}^{k-1} \tau_{i}\left(c_{j}^{ \pm}\right) t^{i}, \quad M_{k}\left(c_{j}^{ \pm}\right)=\sum_{\substack{i=1 \\
S_{i} \text { or } \widehat{S_{i}}=M}}^{k-1} \tau_{i}\left(c_{j}^{ \pm}\right) t^{i} \\
\text { and } R_{k}\left(c_{j}^{ \pm}\right)=\sum_{\substack{i=1 \\
S_{i} \text { or } \widehat{S_{i}}=R}}^{k-1} \tau_{i}\left(c_{j}^{ \pm}\right) t^{i} \quad \text { with } j=1,2 .
\end{gathered}
$$

We notice that

$$
\begin{aligned}
& L_{p}\left(c_{1}^{ \pm}\right)=R_{p}\left(c_{2}^{\mp}\right), \quad M_{p}\left(c_{1}^{ \pm}\right)=M_{p}\left(c_{2}^{\mp}\right), \quad R_{p}\left(c_{1}^{ \pm}\right)=L_{p}\left(c_{2}^{\mp}\right), \\
& L_{p}\left(c_{1}^{ \pm}\right)=-L_{p}\left(c_{1}^{\mp}\right), \quad M_{p}\left(c_{1}^{ \pm}\right)=-M_{p}\left(c_{1}^{\mp}\right), \quad \text { and } \quad R_{p}\left(c_{1}^{ \pm}\right)=-R_{p}\left(c_{1}^{\mp}\right) .
\end{aligned}
$$

Separating the terms associated with the symbols $L, M$ and $R$ of the alphabet $\mathscr{A}=\{A, L, M, B, R\}$, the kneading increments for the points $c_{1}$ and $c_{2}$ are

$$
v_{c_{1}}(t)=\left(\frac{L_{p}\left(c_{1}^{+}\right)}{1-\tau_{p}\left(c_{1}^{+}\right) t^{p}}-\frac{1-L_{p}\left(c_{1}^{+}\right)}{1+\tau_{p}\left(c_{1}^{+}\right) t^{p}}\right) L+\left(\frac{1+M_{p}\left(c_{1}^{+}\right)}{1-\tau_{p}\left(c_{1}^{+}\right) t^{p}}-\frac{-M_{p}\left(c_{1}^{+}\right)}{1+\tau_{p}\left(c_{1}^{+}\right) t^{p}}\right) M+\left(\frac{R_{p}\left(c_{1}^{+}\right)}{1-\tau_{p}\left(c_{1}^{+}\right) t^{p}}-\frac{-R_{p}\left(c_{1}^{+}\right)}{1+\tau_{p}\left(c_{1}^{+}\right) t^{p}}\right) R
$$

and

$$
v_{c_{2}}(t)=\left(\frac{-R_{p}\left(c_{1}^{+}\right)}{1+\tau_{p}\left(c_{1}^{+}\right) t^{p}}-\frac{R_{p}\left(c_{1}^{+}\right)}{1-\tau_{p}\left(c_{1}^{+}\right) t^{p}}\right) L+\left(\frac{-M_{p}\left(c_{1}^{+}\right)}{1+\tau_{p}\left(c_{1}^{+}\right) t^{p}}-\frac{1+M_{p}\left(c_{1}^{+}\right)}{1-\tau_{p}\left(c_{1}^{+}\right) t^{p}}\right) M+\left(\frac{1-L_{p}\left(c_{1}^{+}\right)}{1+\tau_{p}\left(c_{1}^{+}\right) t^{p}}-\frac{L_{p}\left(c_{1}^{+}\right)}{1-\tau_{p}\left(c_{1}^{+}\right) t^{p}}\right) R .
$$

So, we have $N_{23}(t)=-N_{11}(t), N_{22}(t)=-N_{12}(t)$ and $N_{21}(t)=-N_{13}(t)$, and consequently,

$$
\begin{aligned}
& D_{1}(t)=N_{12}(t) N_{23}(t)-N_{13}(t) N_{22}(t)=N_{12}(t)\left(N_{13}(t)-N_{11}(t)\right), \\
& D_{2}(t)=N_{11}(t) N_{23}(t)-N_{13}(t) N_{21}(t)=\left(N_{13}(t)+N_{11}(t)\right)\left(N_{13}(t)-N_{11}(t)\right), \\
& D_{3}(t)=N_{11}(t) N_{22}(t)-N_{12}(t) N_{23}(t)=N_{12}(t)\left(N_{13}(t)-N_{11}(t)\right) .
\end{aligned}
$$

For the particular case of bistable symmetric bimodal maps, we get a similar result. 
Lemma 6. Let $S=\left(A S_{1} S_{2} \cdots S_{p-1} B \widehat{S}_{1} \widehat{S}_{2} \cdots \widehat{S}_{p-1}\right)^{\infty}$ be a symmetric bimodal kneading sequence with period $q=2 p$, satisfying the mirror transformation (12). Then, we have

$$
D_{1}(t)=D_{3}(t)=N_{12}(t)\left(N_{13}(t)-N_{11}(t)\right) \quad \text { and } \quad D_{2}(t)=N_{13}^{2}(t)-N_{11}^{2}(t) \text {. }
$$

Proof. The kneading matrix $N(t)$ has entries obtained from $v_{c_{j}}(t)(5)$ and $v_{c_{j}}(t)$ is defined by (4). Set

$$
\begin{aligned}
& c_{1}^{+} \rightarrow\left(M S_{1} S_{2} \cdots S_{p-1} M \widehat{S}_{1} \widehat{S}_{2} \cdots \widehat{S}_{p-1}\right)^{\infty} \quad \text { and } \quad c_{1}^{-} \rightarrow\left(L S_{1} S_{2} \cdots S_{p-1} R \widehat{S}_{1} \widehat{S}_{2} \cdots \widehat{S}_{p-1}\right)^{\infty}, \\
& c_{2}^{+} \rightarrow\left(R \widehat{S}_{1} \widehat{S}_{2} \cdots \widehat{S}_{p-1} L S_{1} S_{2} \cdots S_{p-1}\right)^{\infty} \quad \text { and } \quad c_{2}^{-} \rightarrow\left(M \widehat{S}_{1} \widehat{S}_{2} \cdots \widehat{S}_{p-1} M S_{1} S_{2} \cdots S_{p-1}\right)^{\infty}
\end{aligned}
$$

Note that, due to the symmetry of the map, the right side of $A(M)$, is the mirror of the left side of $B(M)$ and the left side of $A(L)$, is the mirror of the right side of $B(R)$. The mirror of $S_{k}$ is $\widehat{S}_{k}$, for all $k=1, \ldots, p-1$. Thus, we have

$$
\theta_{c_{1}^{+}}(t)=\left(M+\sum_{k=1}^{k=p-1} \tau_{k}\left(c_{1}^{+}\right) t^{k} S_{k}+\tau_{p}\left(c_{1}^{+}\right) t^{p} M+\sum_{k=1}^{k=p-1} \tau_{p+k}\left(c_{1}^{+}\right) t^{p+k} \widehat{S}_{k}\right) \frac{1}{1-t^{2 p}}
$$

and

$$
\theta_{c_{1}^{-}}(t)=\left(L+\sum_{k=1}^{k=p-1} \tau_{k}\left(c_{1}^{-}\right) t^{k} S_{k}+\tau_{p}\left(c_{1}^{-}\right) t^{p} R+\sum_{k=1}^{k=p-1} \tau_{p+k}\left(c_{1}^{-}\right) t^{p+k} \widehat{S}_{k}\right) \frac{1}{1-t^{2 p}}
$$

Attending to the periodicity of $S$, these formal power series are geometric series and have a common positive ratio $t^{2 p}$, because the number of symbols $L, M$ and $R$ are even in each sequence of $2 p$ consecutive terms of the series. Denote by

$$
L_{k}\left(c_{j}^{ \pm}\right)=\sum_{\substack{i=1 \\ S_{i}=L}}^{k-1} \tau_{i}\left(c_{j}^{ \pm}\right) t^{i}, \quad M_{k}\left(c_{j}^{ \pm}\right)=\sum_{\substack{i=1 \\ S_{i}=M}}^{k-1} \tau_{i}\left(c_{j}^{ \pm}\right) t^{i}
$$

and

$$
R_{k}\left(c_{j}^{ \pm}\right)=\sum_{\substack{i=1 \\ S_{i}=R}}^{k-1} \tau_{i}\left(c_{j}^{ \pm}\right) t^{i} \quad \text { with } j=1,2 .
$$

Looking to the first $p$ symbols of the sequences of $c_{j}^{+}$and $c_{j}^{-}$, we see that the only difference is in $S_{0}$, which have opposite signs, so we have, $\tau_{k}\left(c_{j}^{+}\right)=-\tau_{k}\left(c_{j}^{-}\right)$, for $0<k<p$, and $j=1,2$. Analogously, we see that the difference between the complete sequences of $c_{j}^{+}$and $c_{j}^{-}$is in $S_{0}$ and $S_{p}$, which have the same sign, so we have, $\tau_{p+k}\left(c_{j}^{+}\right)=\tau_{p+k}\left(c_{j}^{-}\right)$. This implies that

$$
\begin{aligned}
& L_{p}\left(c_{j}^{+}\right)=\sum_{\substack{i=1 \\
S_{i}=L}}^{p-1} \tau_{i}\left(c_{j}^{+}\right) t^{i}=-\sum_{\substack{i=1 \\
S_{i}=L}}^{p-1} \tau_{i}\left(c_{j}^{-}\right) t^{i}=-L_{p}\left(c_{j}^{-}\right), \\
& L_{q-p}\left(c_{j}^{+}\right)=\sum_{\substack{i=1 \\
S_{i}=L}}^{p-1} \tau_{p+i}\left(c_{j}^{+}\right) t^{p+i}=\sum_{\substack{i=1 \\
S_{i}=L}}^{p-1} \tau_{p+i}\left(c_{j}^{-}\right) t^{p+i}=L_{q-p}\left(c_{j}^{-}\right), \\
& M_{p}\left(c_{j}^{+}\right)=-M_{p}\left(c_{j}^{-}\right), \quad M_{q-p}\left(c_{j}^{+}\right)=M_{q-p}\left(c_{j}^{-}\right), \\
& R_{p}\left(c_{j}^{+}\right)=-R_{p}\left(c_{j}^{-}\right) \text {and } R_{q-p}\left(c_{j}^{+}\right)=R_{q-p}\left(c_{j}^{-}\right) \quad \text { with } j=1,2 .
\end{aligned}
$$

So, the kneading increment is

$$
v_{c_{1}}(t)=\left\{\left[-1+2 L_{p}\left(c_{1}^{+}\right)\right] L+\left[1+\tau_{p}\left(c_{1}^{+}\right) t^{p}+2 M_{p}\left(c_{1}^{+}\right)\right] M+\left[-\tau_{p}\left(c_{1}^{-}\right) t^{p}+2 R_{p}\left(c_{1}^{+}\right)\right] R\right\} \frac{1}{1-t^{2 p}} .
$$

Notice that, due to $\varepsilon\left(S_{i}\right)=\varepsilon\left(\widehat{S}_{i}\right)$, for all $i$, we have

$$
\tau_{p}\left(c_{1}^{-}\right)=\tau_{p}\left(c_{2}^{+}\right) \text {and } \tau_{p}\left(c_{1}^{+}\right)=\tau_{p}\left(c_{2}^{-}\right),
$$

consequently,

$$
L_{p}\left(c_{2}^{+}\right)=-R_{p}\left(c_{1}^{+}\right), \quad M_{p}\left(c_{2}^{+}\right)=-M_{p}\left(c_{1}^{+}\right) \quad \text { and } \quad R_{p}\left(c_{2}^{+}\right)=-L_{p}\left(c_{1}^{+}\right) .
$$

In the same way, we compute the invariant coordinates and the kneading increment of $c_{2}$ and by equalities (14), we may write

$$
v_{c_{2}}(t)=\left\{\left[\tau_{p}\left(c_{1}^{-}\right) t^{p}-2 R_{p}\left(c_{1}^{+}\right)\right] L+\left[-1-\tau_{p}\left(c_{1}^{+}\right) t^{p}-2 M_{p}\left(c_{1}^{+}\right)\right] M+\left[1-2 L_{p}\left(c_{1}^{+}\right)\right] R\right\} \frac{1}{1-t^{2 p}} .
$$


From (13) and (15), it follows immediately the kneading matrix and the desired result.

As a consequence of the above results, we have

Proposition 7. Let $f_{a, b}$ be a symmetric bimodal map of type (10) or (11) in the sense of Definition 4 and $D(t)$ the kneading determinant (7). Let $s=1 / t^{*}$ be the growth number of $f_{a, b}$, where

$$
t^{*}=\min \left\{t \in[0,1]: N_{13}(t)-N_{11}(t)=0\right\} .
$$

Then, the topological entropy of the map $f_{a, b}$ is $\log s$.

Proof. Considering (8) and by formula (7), the roots of $D(t)$ are also the roots of $D_{1}(t), D_{2}(t)$ and $D_{3}(t)$. From Lemmas 5 and 6 , we have

$$
D_{1}(t)=D_{3}(t)=N_{12}(t)\left[N_{13}(t)-N_{11}(t)\right]
$$

and

$$
D_{2}(t)=\left[N_{13}(t)+N_{11}(t)\right]\left[N_{13}(t)-N_{11}(t)\right] .
$$

So, the smaller real positive root of $D(t)$ occurs when the common factor of $D_{1}(t), D_{2}(t)$ and $D_{3}(t)$ vanish.

Notice that, although the sequence $S$ has $2 p$ terms, it suffices the first $p$ terms to determine its dynamics and the symbols $M$ does not matter in the evaluation of the topological entropy. This suggests that the behaviour of a symmetric bimodal map is determined by some unimodal map, as pointed out in [2].

\section{Duffing application}

Example 8. Let us take the Duffing equation (1) with the parameter values $a=0.2954$ and $b=0.2875$. In this case, the attractor and the unimodal Poincaré return map are shown in Fig. 3. The symbolic sequence is $(C R L R R R)^{\infty}$, so we have

$$
c^{+} \rightarrow(R R L R R R)^{\infty} \text { and } c^{-} \rightarrow(\text { LRLRRR })^{\infty} \text {. }
$$

The invariant coordinates of the sequence $S$ associated with the critical point $c$ are

$$
\begin{aligned}
& \theta_{c^{+}}(t)=\frac{t^{2}}{1+t^{6}} L+\frac{1-t+t^{3}-t^{4}+t^{5}}{1+t^{6}} R, \\
& \theta_{c^{-}}(t)=\frac{1-t^{2}}{1-t^{6}} L+\frac{t-t^{3}+t^{4}-t^{5}}{1-t^{6}} R .
\end{aligned}
$$

The kneading increment of the critical point, $v_{c}(t)=\theta_{c^{+}}(t)-\theta_{c^{-}}(t)$, is

$$
v_{c}(t)=\frac{-1+2 t^{2}-t^{6}}{1-t^{12}} L+\frac{1-2 t+2 t^{3}-2 t^{4}+2 t^{5}-t^{6}}{1-t^{12}} R
$$

So, the kneading matrix is $N(t)=\left[\begin{array}{ll}N_{11}(t) & N_{12}(t)\end{array}\right]=\left[\begin{array}{ll}D_{2}(t) & D_{1}(t)\end{array}\right]$, i.e.,

$$
N(t)=\left[\begin{array}{ll}
\frac{-1+2 t^{2}-t^{6}}{1-t^{12}} & \frac{1-2 t+2 t^{3}-2 t^{4}+2 t^{5}-t^{6}}{1-t^{12}}
\end{array}\right]
$$

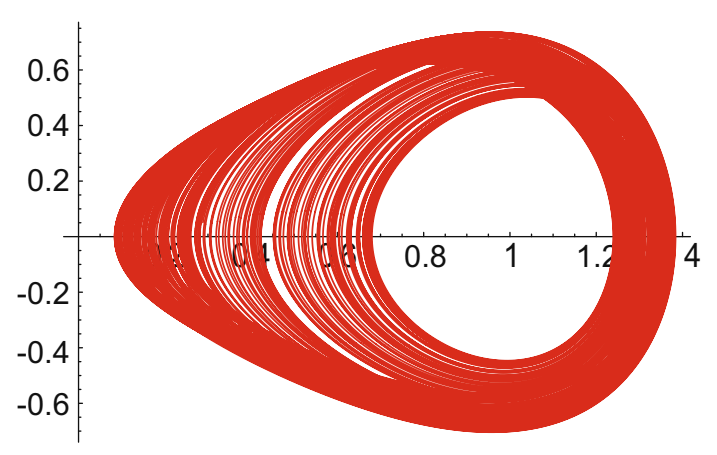

Fig. 3. Duffing attractor and Poincaré return map for $a=0.2954$ and $b=0.2875$.

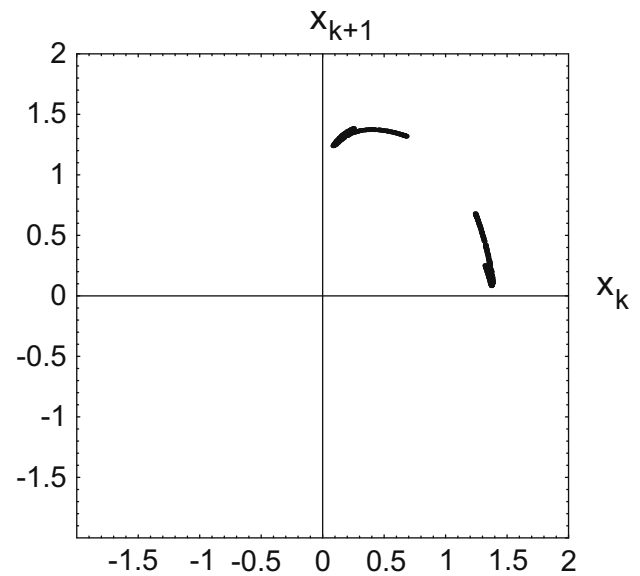



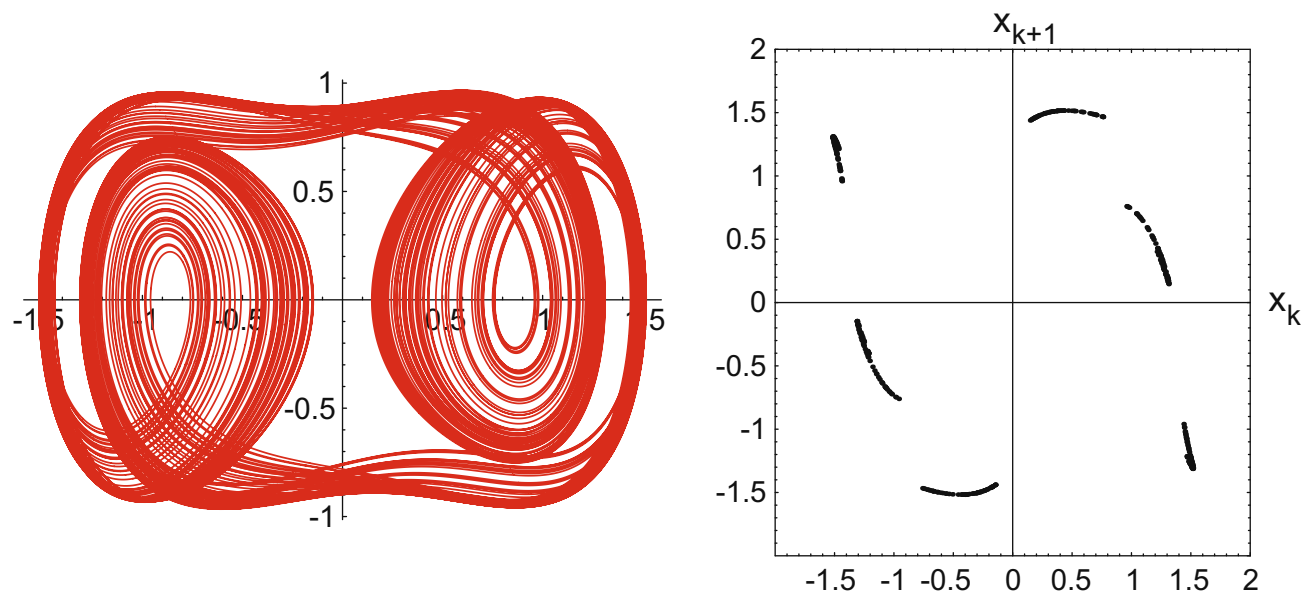

Fig. 4. Duffing attractor and Poincaré return map for $a=0.5$ and $b=0.719$.

and the kneading determinant is

$$
D(t)=\frac{(-1+t)\left(-1+t^{2}+t^{4}\right)}{1-t^{12}} .
$$

The smallest positive real root of $D_{1}(t)$ is $t^{*}=0.786 \ldots$, so the growth number is $s=1 / t^{*}=1.272 \ldots$ and the topological entropy is $h_{\text {top }}=0.2406 \ldots$

Example 9. Let us take, for example, the case $a=0.5$ and $b=0.719$ in the Duffing equation (1). See in Fig. 4 the attractor and the return map, which behaves, in this case, like a symmetric bimodal map. The symbolic sequence is bistable and symmetric $(\text { ALRMRLLLRBRLMLRRRL })^{\infty}$. Thus we have

$$
\begin{aligned}
& c_{1}^{+} \rightarrow(\text { MLRMRLLLRMRLMLRRRL })^{\infty}, \quad c_{1}^{-} \rightarrow(\text { LLRMRLLLRRLMLRRRL })^{\infty}, \\
& c_{2}^{+} \rightarrow(\text { RRLMLRRRLLLRMRLLLR })^{\infty}, \quad c_{2}^{-} \rightarrow(M R L M L R R R L M L R M R L L L R)^{\infty} .
\end{aligned}
$$

Computing the invariant coordinates $\theta_{c_{i}^{ \pm}}(t)$ and the kneading increments $v_{c_{i}^{ \pm}}(t)(i=1,2)$, we obtain the kneading matrix, from which we have

$$
D_{1}(t)=D_{3}(t)=\frac{(1+t)^{2}\left(1-3 t+t^{2}\right)\left(1-t+t^{2}\right)\left(-1-t^{3}+t^{6}\right)\left(-1+t^{3}+t^{6}\right)}{1-t^{18}} .
$$

From $D_{1}(t)=0$, we get $t=0.381966 \ldots, s=2.61803 \ldots$ and $h_{\text {top }}(f)=0.962424 \ldots$

See in Table 3, the kneading data associated to symmetric bimodal maps $f_{a, b}$ for some values of $(a, b) \in \mathscr{B}$ and $w=1.18$. For all these examples, we have chaotic behaviour and the topological entropy has exactly the same value 0.962424 ...

\section{References}

[1] Duffing G. Erzwungene Schwingungen bei veränderlicher Eigenfrequenz und ihre Technische Beduetung. Vieweg Braunschweig 1918 .

[2] Xie Fa-Gen, Zheng Wei-Mou, Hao Bai-Lin. Symbolic dynamics of the two-well Duffing equation. Commun Theor Phys 1995;24:43-52.

[3] Jing Z, Huang J, Deng J. Complex dynamics in three-well Duffing system with two external forcings. Chaos, Solitons \& Fractals 2007;33:795-812.

[4] Khammari H, Mira C, Carcassés J-P. Behaviour of harmonics generated by a Duffing type equation with a nonlinear damping. Part I. Int J Bifurc Chaos 2005;15(10):3181-221.

[5] Lampreia JP, Sousa Ramos J. Symbolic dynamics for bimodal maps. Portugaliae Math 1997;54(1):1-18.

[6] Leonel Rocha J, Sousa Ramos J. Weighted kneading theory of one-dimensional maps with a hole. Int J Math Math Sci 2004;38:2019-38.

[7] Leonel Rocha J, Sousa Ramos J. Computing conditionally invariant measures and escape rates. Neural Parallel Sci Comput 2006;14:97-114.

[8] Milnor J, Thurston W. On iterated maps of the interval. Lect notes in math, vol. 1342. Berlin: Springer; 1988 . p. 465-563.

[9] Mira C, Touzani-Qriquet M, Kawakami H. Bifurcation structures generated by the nonautonomous Duffing equation. Int J Bifurc Chaos 1999;9(7):1363-79.

[10] Misiurewicz M, Szlenk W. Entropy of piecewise monotone mappings. Studia Math 1980;67:45-63.

[11] El Naschie MS. Hierarchy of kissing numbers for exceptional Lie symmetry groups in high energy physics. Chaos, Solitons \& Fractals 2008;35:420-2.

[12] El Naschie MS. A derivation of the fine structure constant from the exceptional Lie group hierarchy of the micro cosmos. Chaos, Solitons \& Fractals 2008;36:819-22.

[13] Njah AN, Vincent UE. Chaos synchronization between single and double wells Duffing-Van der Pol oscillators using active control. Chaos, Solitons \& Fractals 2008;37:1356-61.

[14] Wu X, Cai J, Wang M. Global chaos synchronization of the parametrically excited Duffing oscillators by linear state error feedback control. Chaos, Solitons \& Fractals 2008;36:121-8.

[15] Zeeman EC. Duffing's equation in brain modeling. J Inst Math Appl 1976:207-14. 